\title{
Obesity contributes more to increasing ApoB/ApoA1 ratio than hyperandrogenism in PCOS women aged 20-38 years in China
}

\author{
JIANHUA ZHENG $^{1 *}$, QIANQIAN YIN ${ }^{2 *}$, JUANYI CAO $^{2}$ and BEI ZHANG ${ }^{1}$ \\ ${ }^{1}$ Department of Gynecology and Obstetrics, and ${ }^{2}$ Center for Reproductive Medicine, \\ Xuzhou Central Hospital Affiliated to Southeast University, Xuzhou, Jiangsu 221009, P.R. China
}

Received June 28, 2016; Accepted January 3, 2017

DOI: $10.3892 /$ etm.2017.4094

\begin{abstract}
We investigated the association between the apolipoprotein B (ApoB)/apolipoprotein A1 (ApoA1) ratio and insulin resistance (IR), metabolic syndrome (MS) and its components in Chinese polycystic ovary syndrome (PCOS) adults. We also examined whether hyperandrogenism is involved in obesityrelated metabolic abnormalities in a cohort of patients. A clinical cross-sectional study consisting of 532 Chinese PCOS adults aged 20-38 years was designed. Each subject underwent a physical examination and laboratory evaluation. We found that the ApoB/ApoA1 ratio was significantly higher in patients with MS compared to those without MS. This test provided $83.6 \%$ sensitivity and $67.6 \%$ specificity with a threshold value of 0.60 in MS. The ApoB/ApoA1 ratio increased significantly as the number of MS components increased. After adjusting for age, the patients with MS or IR were more likely to be in the high ApoB/ApoA1 group, and the ApoB/ApoA1 ratio was associated significantly with each of the MS components, high free testosterone (FT), and high free androgen index (FAI). After adjusting for age and body mass index (BMI), the association persisted for all factors excluding high blood pressure (BP), high fasting plasma glucose (FPG), high FT, and high FAI. Therefore, the ApoB/ApoA1 ratio was strongly associated with IR, MS and its components, high FT, and high FAI. A high ApoB/ApoA1 ratio appears to be a good predictive marker of MS in Chinese PCOS adults. Obesity, especially central obesity, contributes more to increasing ApoB/ApoA1 ratio than increased $\mathrm{BP}, \mathrm{FPG}, \mathrm{FT}$ and FAI in this cohort of PCOS patients.
\end{abstract}

Correspondence to: Dr Bei Zhang, Department of Gynecology and Obstetrics, Xuzhou Central Hospital Affiliated to Southeast University, 199 Jiefang Road, Xuzhou, Jiangsu 221009, P.R. China E-mail: bei_zhang111@163.com

*Contributed equally

Key words: apolipoprotein B, apolipoprotein A1, ApoB/ApoA1 ratio, metabolic syndrome, insulin resistance, free testosterone, free testosterone index, polycystic ovary syndrome

\section{Introduction}

Apolipoprotein B (ApoB) is the total amount of potentially atherogenic circulating compounds, including low-density lipoproteins (LDLs). ApoB is easily oxidized, induces an inflammatory reaction and leads to the formation of plaques in the arterial wall. Apolipoprotein A1 (ApoA1) is the major component of high-density lipoprotein (HDL) particles and plays a prominent role in the reverse transportation of cholesterol, as well as some anti-inflammatory and antioxidant processes. Therefore, the $\mathrm{ApoB} / \mathrm{ApoA} 1$ ratio reflects the status of pro- and anti-atherogenic lipoproteins and may serve as a good marker for assessing cardiovascular disease (CVD) risk (1-3).

The polycystic ovary syndrome (PCOS), which is characterized by chronic ovulatory dysfunction, hyperandrogenism, polycystic ovary morphology, obesity, and insulin resistance (IR), is a common endocrine disorder affecting 5-10\% of women of reproductive age (4). Patients with PCOS are at an increased risk of developing metabolic syndrome (MS) (5). MS is a cluster of metabolic abnormalities that place individuals at increased risk of CVD and is associated with IR.

Results from previous studies indicated that the ApoB/ApoA1 ratio is superior to any traditional cholesterol ratios, lipids and lipoproteins for the purpose of predicting CVD in populations of different races, gender and ages (6-8). However, to the best of our knowledge, studies on the use of ApoB/ApoA1 ratio in PCOS women are limited. Yin et al reported that the ApoB/ApoA1 ratio was a good predictive marker of MS and that the free androgen index (FAI) could be involved in obesity-related metabolic abnormalities in Chinese female adolescents with PCOS (9). However, it is unclear whether the results are similar in PCOS adults in China. Therefore, the aim of this study was to assess the association between the ApoB/ApoA1 ratio and IR, MS and its components in Chinese PCOS adults. We also wanted to investigate whether hyperandrogenism is involved in obesityrelated metabolic abnormalities in this cohort of patients.

\section{Patients and methods}

Patient selection. A total of 532 PCOS patients, between the ages of 20 and 38 years, with complete medical records were recruited from the Gynecological Outpatient Department of 
Xuzhou Central Hospital Affiliated to Southeast University from August 2009 to April 2015. Any medications known to affect sex hormones and glucose or lipid metabolism, with the exception of oral contraceptives, were discontinued for at least 1 month to 3 months before the study. None of the patients used any hypolipidemic or anti-hypertensive drugs. The study was approved by the Institutional Review Board of Xuzhou Central Hospital. Informed consent was obtained from each patient.

PCOS diagnosis. PCOS patients were diagnosed according to the Rotterdam criteria (10) which required the meeting of at least two out of the following three criteria: i) Oligo- and/or anovulation (i.e., $\leq 8$ menstrual periods in a year or menstrual cycles $>35$ days in length) (11); ii) clinical hyperandrogenism [i.e., acne or modified Ferriman-Gallwey scores $\geq 8$ (12)] or biochemical hyperandrogenism (i.e., serum total testosterone (TT) $\geq 2.6 \mathrm{nmol} / 1$, free testosterone (FT) $\geq 6.0 \mathrm{pg} / \mathrm{ml}$, TT and FT normal values were determined by the Clinical Laboratory of the Gynecology Department at Xuzhou Central Hospital); and iii) ultrasonographic findings of ovarian polycystic morphology (i.e., presence of $\geq 12$ follicles in each ovary measuring 2-9 $\mathrm{mm}$ in diameter) and exclusion of related disorders such as hypothyroidism, hyperprolactinemia, and adrenal hyperplasia by both physical examination and lab tests for thyroid stimulating hormone (TSH), prolactin (PRL), and serum 17a-hydroxyprogesterone (17a-OHP).

Patients were diagnosed with MS based on International Diabetes Federation (IDF) criteria for MS (13). The guideline for MS diagnosis included the presence of central obesity [waist circumference (WC), $\geq 80 \mathrm{~cm}$ ] plus two or more of the following four factors: i) Increased concentration of triglycerides (TG): $\geq 1.7 \mathrm{mmol} / \mathrm{l}$; ii) reduced concentration of HDL cholesterol (HDL-c) $<1.29 \mathrm{mmol} / \mathrm{l}$; iii) raised blood pressure (BP): systolic BP (SBP) $\geq 130 \mathrm{mmHg}$ or diastolic BP (DBP) $\geq 85 \mathrm{mmHg}$ or treatment of previously diagnosed hypertension (HBP); and iv) increased fasting plasma glucose (FPG) level $\geq 5.6 \mathrm{mmol} / 1$ or previously diagnosed type 2 diabetes mellitus (T2DM).

Study protocol. All the PCOS patients underwent anthropometric measurements including weight, height, waist and hip circumferences, BP, modified Ferriman-Gallwey scores, and acne scores. Ferriman-Gallwey scores were assessed by at least two observers. Body weight, height, and waist and hip circumferences were measured based on methods recommended by the World Health Organization (14). Body mass index (BMI) was calculated as weight $(\mathrm{kg}) /$ height $\left(\mathrm{m}^{2}\right)$.

Fasting blood samples were obtained from PCOS patients starting from the first to fifth day of the menstrual period/withdrawal bleed in order to measure PRL and TT by the chemiluminescence immunoassays (Beckman Coulter, Fullerton, CA, USA). FT, sex hormone-binding globulin (SHBG), dehydroepiandrosterone sulfate (DHEAS), and 17a-OHP were measured by Access 2 ELISA (Beckman Coulter). TSH was measured using a chemiluminescence immunometric assay (Immulite 2000 Analyzer; CPC, Los Angeles, CA, USA). The FAI, a parameter of bioavailable testosterone, was calculated from TT and SHBG as follows: $\mathrm{FAI}=[\mathrm{TT}(\mathrm{nmol} / \mathrm{l}) \times 100 / \mathrm{SHBG}(\mathrm{nmol} / \mathrm{l})]$. Fasting venous blood samples were also used to measure the levels of high
FPG, insulin, total cholesterol (CHOL), TG, HDL-c and LDL cholesterol (LDL-c). Plasma glucose was measured by the glucose oxidase method (Hitachi 7600; Hitachi, Tokyo, Japan) and plasma insulin was measured using a chemiluminescence immunometric assay and commercial kit (Immulite 2000 Analyzer; CPC). CHOL, HDL-c, LDL-c and TG were measured using an enzymatic calorimetric method with the 7600 autoanalyzer (Hitachi 7600). ApoB and ApoA1 were measured by the immunoturbidimetric method (Hitachi 7600). Insulin sensitivity was estimated by the homeostasis model assessment (HOMA-IR): [fasting insulin (mU/l) x FPG $(\mathrm{mmol} / \mathrm{l})] / 22.5$, and IR was defined as HOMA-IR $\geq 2.14$ (15). The high ApoB/ApoA1, high FT and high FAI groups were defined as the 75th of the ApoB/ApoA1 ratio $(\geq 0.68)$, FT levels $(\geq 4.86)$ and FAI levels $(\geq 7.38)$, respectively, and the remaining three quartiles were merged into the low ApoB/ApoA1, low FT and low FAI groups, respectively.

Statistical analysis. The data were analyzed using the statistical software SPSS version 13.0 for Windows (SPSS, Inc., Chicago, IL, USA). We assessed the normality of the distribution of all continuous variables using the Kolmogorov-Smirnov test. Since the data were not normally distributed, continuous variables were presented as median values with an interquartile range (25-75th percentile), and categorical variables were described as a percentage. Differences in medians between groups were determined by the Mann-Whitney U test. For categorical variables, the Pearson $\chi^{2}$ test was used to analyze the differences between groups. Receiver operating characteristic (ROC) curve analysis was used to evaluate the predictive value for the ApoB/ApoA1 ratio to predict MS. The association between the ApoB/ApoA1 ratio and characteristics of the study population were tested by Spearman's rank correlations analysis. Adjusted binary logistic regression analysis was used to estimate whether the patients with the IR, MS and its components, high FT and high FAI were at a risk of being in the high $\mathrm{ApoB} / \mathrm{ApoA} 1$ group. $\mathrm{P}<0.05$ (two-tailed) was considered statistically significant.

\section{Results}

General characteristics and ROC curve. Of all the 532 subjects, 73 subjects met the criteria for the diagnosis of MS. The cohort included 19 subjects with T2DM and 84 subjects with hypertension. There were no statistically significant differences with regards to the levels of TT and DHEAS between the PCOS patients in the high and low ApoB/ApoA1 groups. The mean age, WC, SBP, DBP, FPG, CHOL, TG, LDL-c, HOMA-IR, FT and FAI levels were higher and HDL-c and SHBG levels were lower in the high ApoB/ApoA1 group when compared to the low ApoB/ApoA1 group. Similar results were obtained when the parameters were detected between the PCOS patients without and with MS. The percentage of patients who met the criteria for T2DM, HBP, IR, and MS were significantly greater in the high ApoB/ApoA1 group than in the low ApoB/ApoA1 group (Table I).

The ApoB/ApoA1 ratio provided $83.6 \%$ sensitivity and $67.6 \%$ specificity with a threshold value of 0.60 in MS, the area under the ROC curve was 0.823 (95\% CI=0.778-0.869), and the Youden index was 0.51 (Fig. 1). 
Table I. General characteristics of PCOS patients.

\begin{tabular}{|c|c|c|c|c|}
\hline Characteristics & $\begin{array}{l}\text { Low ApoB/Apo1 } \\
\qquad(n=399)\end{array}$ & $\begin{array}{l}\text { High ApoB/ApoA1 } \\
\qquad(\mathrm{n}=133)\end{array}$ & $\begin{array}{l}\text { Without MS } \\
\quad(n=459)\end{array}$ & $\begin{array}{l}\text { With MS } \\
(\mathrm{n}=73)\end{array}$ \\
\hline Age (years) & $26(22-29)^{\mathrm{a}}$ & $27(25-30)$ & $26.0(22.0-29.0)^{\mathrm{b}}$ & $29.0(25.3-30.8)$ \\
\hline $\mathrm{BMI}\left(\mathrm{kg} / \mathrm{m}^{2}\right)$ & $20.96(19.04-23.88)^{\mathrm{a}}$ & $25.30(21.76-28.08)$ & $21.1(19.1-23.5)^{\mathrm{b}}$ & $28.0(26.0-30.5)$ \\
\hline $\mathrm{WC}(\mathrm{cm})$ & $73(67-79)^{\mathrm{a}}$ & $82(74-90)$ & $73.0(68.0-78.0)^{\mathrm{b}}$ & $88.8(86.0-97.8)$ \\
\hline $\mathrm{SBP}(\mathrm{mmHg})$ & $111(104-120)^{\mathrm{a}}$ & $117(105-124)$ & $110(101-120)^{\mathrm{b}}$ & 125 (119-133) \\
\hline DBP (mmHg) & $72.0(68.0-78.5)^{\mathrm{a}}$ & $78(70-82)$ & $70(68-78)^{\mathrm{b}}$ & $81(75-87)$ \\
\hline FPG (mmol/l) & $5.0(4.7-5.3)^{\mathrm{a}}$ & $5.1(4.9-5.6)$ & $5.0(4.7-5.3)^{\mathrm{b}}$ & $5.6(5.0-6.0)$ \\
\hline CHOL (mmol/l) & $4.74(4.30-5.31)^{\mathrm{a}}$ & $5.27(4.63-5.91)$ & $4.79(4.36-5.37)^{\mathrm{b}}$ & $5.31(4.66-6.07)$ \\
\hline $\mathrm{TG}(\mathrm{mmol} / \mathrm{l})$ & $0.97(0.73-1.32)^{\mathrm{a}}$ & $1.59(1.13-2.18)$ & $1.03(0.74-1.35)^{\mathrm{b}}$ & $2.14(1.52-3.03)$ \\
\hline HDL-c (mmol/l) & $1.62(1.41-1.85)^{\mathrm{a}}$ & $1.18(1.05-1.37)$ & $1.57(1.36-1.80)^{\mathrm{b}}$ & $1.14(1.03-1.27)$ \\
\hline LDL-c (mmol/l) & $2.67(2.26-3.17)^{\mathrm{a}}$ & 3.35 (2.87-3.97) & $2.81(2.35-3.34)^{\mathrm{b}}$ & $3.22(2.78-3.95)$ \\
\hline APOB/APOA1 ratio & - & - & $0.51(0.43-0.63)^{\mathrm{b}}$ & $0.76(0.63-0.93)$ \\
\hline $\mathrm{TT}(\mathrm{nmol} / \mathrm{l})$ & $2.27(1.68-2.94)$ & $2.28(1.72-2.80)$ & $2.29(1.70-2.95)$ & $2.07(1.67-2.72)$ \\
\hline SHBG (nmol/l) & $58.83(35.93-89.84)^{\mathrm{a}}$ & $37.85(24.42-58.79)$ & $56.9(30.1-87.2)^{\mathrm{b}}$ & $31.2(19.9-44.6)$ \\
\hline $\mathrm{FT}(\mathrm{pg} / \mathrm{ml})$ & $2.88(1.89-4.59)^{\mathrm{a}}$ & $3.35(2.17-6.20)$ & $2.87(1.91-4.58)^{\mathrm{b}}$ & $4.06(2.52-6.87)$ \\
\hline DHEAS (ng/ml) & $1637.5(730.8-2849.7)$ & $1230.0(774.8-2106.0)$ & 1607 (769-2835) & $903(720-2034)$ \\
\hline FAI & $3.90(2.30-6.39)^{\mathrm{a}}$ & $5.92(2.93-8.99)$ & $3.90(2.31-6.19)^{\mathrm{b}}$ & $7.54(4.72-11.30)$ \\
\hline HOMA-IR & $1.51(0.81-2.51)^{\mathrm{a}}$ & $2.76(1.69-4.28)$ & $1.58(0.84-2.45)^{\mathrm{b}}$ & $3.71(2.37-5.95)$ \\
\hline T2DM, n (\%) & $10(2.51)^{\mathrm{a}}$ & $9(0.24)$ & - & - \\
\hline $\mathrm{HBP}, \mathrm{n}(\%)$ & $49(12.28)^{\mathrm{a}}$ & $35(26.32)$ & - & - \\
\hline MS, n (\%) & $25(6.27)^{\mathrm{a}}$ & $48(30.09)$ & - & - \\
\hline IR, n (\%) & $127(31.83)^{\mathrm{a}}$ & $87(65.41)$ & - & - \\
\hline
\end{tabular}

Variables are expressed as median with (25-75th percentile). PCOS, polycystic ovary syndrome; BMI, body mass index; WC, waist circumference; SBP, systolic blood pressure; DBP, diastolic blood pressure; FPG, fasting plasma glucose; CHOL, total cholesterol; TG, triglycerides; HDL-c, high-density lipoprotein cholesterol; LDL-c, low-density lipoprotein cholesterol; ApoB/ApoA1 ratio, apolipoprotein B/apolipoprotein A1 ratio; TT, total testosterone; SHBG, sex hormone-binding globulin; FT, free testosterone; DHEAS, dehydroepiandrosterone sulfate; FAI, free androgen index $=(\mathrm{T} x$ 100)/SHBG; HOMA-IR, homeostasis model assessment of insulin resistance; T2DM, type 2 diabetes mellitus; HBP, hypertension; MS, metabolic syndrome; IR, insulin resistance. Mann-Whitney U test and Pearson $\chi^{2}$ test. ${ }^{a}$ Low ApoB/ApoA1 group vs. high ApoB/APOA1 group $\mathrm{P}<0.05$. ${ }^{\text {bW }}$ ithout vs. with MS group $\mathrm{P}<0.05$.

Spearman's correlations. Spearman's rank correlation coefficients between the ApoB/ApoA1 ratio and characteristics of the study population are shown in Table II. There was a statistically significant positive correlation between the ApoB/ApoA1 ratio and age, BMI, FT, FAI, WC, TG, FPG, SBP and DBP, and a significant negative correlation of the ApoB/ApoA1 ratio with HDL-c. The strongest correlation was found to be between the ApoB/ApoA1 ratio and HDL-c $(r=-0.676)$, and the second strongest was between the ApoB/ ApoA1 ratio and TG $(\mathrm{r}=0.446)$.

Median values and MS. More than half of the patients $(57.89 \%)$ had at least one component of MS, indicating that MS components are quite prevalent in this population. We analyzed the ApoB/ApoA1 ratio in the groups with different numbers of MS components and identified that the median values of the ApoB/ApoA1 ratio increased significantly with the increasing number of MS components. The data were analyzed after exclusion of HDL-c and TG; however, the correlation between the median values of the ApoB/ApoA1 ratio and the number of MS components remained (Tables III and IV).

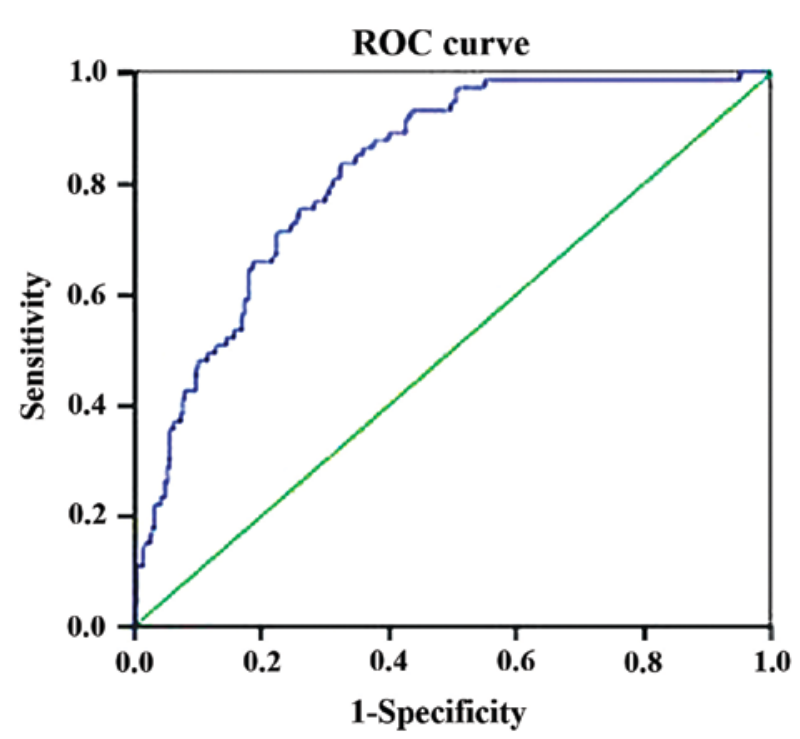

Diagonal segments are produced by ties

Figure 1. ROC curve for ApoB/ApoA1 ratio to predict MS in Chinese PCOS patients. ROC, receiver operating characteristic; ApoB, apolipoprotein B; ApoA1, apolipoprotein A1; MS, metabolic syndrome; PCOS, polycystic ovary syndrome. 
Table II. Spearman's rank correlations between the ApoB/ApoA1 ratio and characteristics of the study population.

\begin{tabular}{lcc}
\hline Variables & $\mathrm{r}$ & P-value \\
\hline Age & 0.181 & $<0.001$ \\
BMI & 0.439 & $<0.001$ \\
FT & 0.179 & $<0.001$ \\
FAI & 0.217 & $<0.001$ \\
SBP & 0.168 & $<0.001$ \\
DBP & 0.231 & $<0.001$ \\
WC & 0.408 & $<0.001$ \\
FPG & 0.184 & $<0.001$ \\
TG & 0.446 & $<0.001$ \\
HDL-c & -0.676 & $<0.001$ \\
\hline
\end{tabular}

ApoB, apolipoprotein B; ApoA1, apolipoprotein A1; BMI, body mass index; FT, free testosterone; FAI, free androgen index $=(\mathrm{T} x$ 100 $) /$ SHBG; SBP, systolic blood pressure; DBP, diastolic blood pressure; DBP, diastolic blood pressure; WC, waist circumference; FPG, fasting plasma glucose; TG, triglycerides; HDL-c, high-density lipoprotein cholesterol. $\mathrm{P}<0.001$, Spearman's rank correlation.

Table III. Median ApoB/ApoA1 ratio of subjects with different numbers of MS components.

\begin{tabular}{lcc}
$\begin{array}{l}\text { No. of } \\
\text { components }\end{array}$ & Case & ApoB/ApoA1 ratio \\
\hline 0 & 224 & $0.48(0.40-0.56)$ \\
1 & 139 & $0.53(0.44-0.65)$ \\
2 & 88 & $0.67(0.56-0.80)$ \\
3 & 49 & $0.70(0.60-0.90)$ \\
4 & 22 & $0.73(0.61-0.86)$ \\
5 & 10 & $0.95(0.66-1.23)$
\end{tabular}

ApoB/ApoA1 is expressed as the median with (25-75th percentile). $\mathrm{P}<0.05$, Mann-Whitney U test. ApoB, apolipoprotein B; ApoA1, apolipoprotein A1; MS, metabolic syndrome.

Logistic regression. We applied adjusted logistic regression models to determine the association between the ApoB/ ApoA1 ratio and the individual components of MS, IR, FT and FAI (Table V). After adjusting for age, patients with MS and IR were 8.13- and 4.22-fold, respectively, more likely to be in the high ApoB/ApoA1 group. The ApoB/ApoA1 ratio was significantly associated with each of the MS components in the following order of descending magnitude: low HDL-c [odds ratio $(\mathrm{OR})=9.89]$, high $\mathrm{TG}(\mathrm{OR}=4.91)$, high $\mathrm{WC}(\mathrm{OR}=4.50)$, high $\mathrm{BP}(\mathrm{OR}=2.36)$, and high $\mathrm{FPG}(\mathrm{OR}=2.04)$. After adjusting for both age and BMI, MS (OR=4.14), IR (OR=2.41), low HDL-c $(\mathrm{OR}=7.29)$, high $\mathrm{TG}(\mathrm{OR}=3.22)$ and high WC $(\mathrm{OR}=1.99)$ was associated significantly with the ApoB/ApoA1 ratio; however, the association was not significant for high BP and high FPG. Patients with high FT and high FAI were 1.85- and 2.39-fold
Table IV. Median ApoB/ApoA1 ratio of subjects with different numbers of MS components after excluding lipid components.

\begin{tabular}{lcc}
$\begin{array}{l}\text { No. of } \\
\text { components }\end{array}$ & Case & ApoB/ApoA1 ratio \\
\hline 0 & 290 & $0.50(0.42-0.61)$ \\
1 & 157 & $0.60(0.46-0.73)$ \\
2 & 63 & $0.64(0.54-0.80)$ \\
3 & 22 & $0.74(0.64-1.05)$ \\
\hline
\end{tabular}

ApoB/ApoA1 ratio is expressed as median with (25-75th percentile). ( $\mathrm{P}<0.05$, Mann-Whitney $\mathrm{U}$ test). ApoB, apolipoprotein $\mathrm{B}$; ApoA1, apolipoprotein A1; MS, metabolic syndrome.

respectively, more likely to be in the high ApoB/ApoA1 group after adjusting for age; the association was not significant after adjusting for age and BMI together. All the parameters provided similar OR values after adjusting for either BMI or WC (data not shown).

\section{Discussion}

In this study, we demonstrated that the ApoB/ApoA1 ratio was significantly higher in patients with IR, and the association between the ratio and HOMA-IR was independent of age and obesity. Sung and Hwang (16), and Sierra-Johnson et al (17) found that an increased ApoB and a decreased ApoB/ApoA1 ratio in normal, non-diabetic subjects may be related to IR in American and Korean subjects, respectively; the results of which were consistent with ours. To explain the association between these two variables, Taghibiglou et al (18), insisted that IR and the hepatic over-production of VLDL-ApoB were closely associated, which led to an increased activity of protein tyrosine phosphatase 1B (PTP-1B). When combined with a decreased activity of cysteine protease, which catalyzes ApoB, this may ultimately increase the hepatic production of VLDL-ApoB (19).

In our study, the ApoB/ApoA1 ratio was associated with MS in Chinese PCOS women aged 20-38 years. The patients with MS have higher ApoB/ApoA1 ratio than those without MS. Compared to the low ApoB/ApoA1 group, the high ApoB/ApoA1 group was more likely to have MS. There have been few studies that have focused on the appropriate cut-off values for MS diagnosis, especially in PCOS adults. We provided the ROC curve of ApoB/ApoA1 ratio for MS in PCOS adults for the first time, and took the ApoB/ApoA1 ratio 0.60 as the cut-off point for MS (with sensitivity $83.6 \%$, specificity $67.6 \%, \mathrm{AUC}=0.82$, and Youden index 0.51 ). Jing et al suggested that an apoB/apoA1 ratio higher than 0.84 in women may be a promising and convenient marker of MS (with sensitivity $65.8 \%$, specificity $68.4 \%$, AUC=0.73) (20). In our study, however, the cut-off values were lower than those reported in previous studies (20). The possible explanation of the discrepancy may lie in the fact that the subjects of our study were all PCOS patients. These results also suggest that a high ApoB/ApoA1 ratio is a good predictive marker of MS in 
Table V. Logistic regression between the ApoB/ApoA1 ratio and individual components of MS, IR, FT and FAI.

\begin{tabular}{|c|c|c|c|c|}
\hline \multirow[b]{2}{*}{ Parameters } & \multicolumn{2}{|c|}{ Age-adjusted } & \multicolumn{2}{|c|}{ Age- and BMI-adjusted } \\
\hline & OR $(95 \% \mathrm{CI})$ & P-value & OR $(95 \% \mathrm{CI})$ & P-value \\
\hline HDL-c $<1.29 \mathrm{mmol} / \mathrm{l}$ & $9.89(6.30-15.52)$ & $<0.05$ & $7.29(4.55-11.69)$ & $<0.05$ \\
\hline $\mathrm{TG} \geq 1.7 \mathrm{mmol} / 1$ & $4.91(3.13-7.71)$ & $<0.05$ & $3.22(1.99-5.21)$ & $<0.05$ \\
\hline $\mathrm{WC}>80 \mathrm{~cm}$ & $4.50(2.94-6.90)$ & $<0.05$ & $1.99(1.07-3.70)$ & $<0.05$ \\
\hline $\mathrm{FPG} \geq 5.6 \mathrm{mmol} / \mathrm{l}$ & $2.04(1.24-3.33)$ & $<0.05$ & $1.38(0.81-2.36)$ & 0.238 \\
\hline $\mathrm{BP} \geq 130 / 85 \mathrm{mmHg}$ & $2.36(1.44-3.88)$ & $<0.05$ & $1.39(0.80-2.41)$ & 0.238 \\
\hline MS & $8.13(4.70-14.05)$ & $<0.05$ & $4.14(2.23-7.69)$ & $<0.05$ \\
\hline IR (HOMA index $\geq 2.14$ ) & $4.22(2.78-6.41)$ & $<0.05$ & $2.41(1.49-3.89)$ & $<0.05$ \\
\hline $\mathrm{FT} \geq 4.86 \mathrm{pg} / \mathrm{ml}$ & $1.85(1.17-2.92)$ & $<0.05$ & $1.26(0.76-2.08)$ & 0.366 \\
\hline $\mathrm{FAI} \geq 7.38$ & $2.39(1.50-3.82)$ & $<0.05$ & $1.31(0.77-2.22)$ & 0.320 \\
\hline
\end{tabular}

$\mathrm{P}<0.05$, adjusted binary logistic regression. ApoB, apolipoprotein $\mathrm{B}$; ApoA1, apolipoprotein A1; MS, metabolic syndrome; IR, insulin resistance; FT, free testosterone; FAI, free androgen index; OR, odds ratio; BMI, body mass index; HDL-c, high-density lipoprotein cholesterol; TG, triglycerides; WC, waist circumference; FPG, fasting plasma glucose; BP, blood pressure.

Chinese PCOS adults, and the predictive value may be slightly lower than it is in adolescent women with PCOS (9).

Moreover, the ApoB/ApoA1 ratio was correlated with all the MS components, especially with HDL-c. The median values of the ApoB/ApoA1 ratio increased as the number of MS components in individual patients increased. Despite the exclusion of TG and HDL-c, the correlation still existed, which indicates that the association between the ApoB/ApoA1 ratio and MS is independent of lipid components.

We also found that MS and its components were quite common in the Chinese PCOS patients $(57.89 \%)$ in this study. A few studies have described that the ApoB/ApoA1 ratio is related to $\mathrm{MS}$ and its components after adjusting for age and gender together (21-24). Our results are in line with the above studies. Nevertheless, none of these studies included only PCOS adults. One study conducted on the civilian population in the USA (mean age, 48 years) showed that subjects with MS were 3.9-fold more likely to be in the 75th quartile of the ApoB/ApoA1 ratio (23). The same study also showed that lower HDL-c levels provided the highest OR in the high ApoB/ApoA1 group, which was consistent with our results. Recently, Zhong et al found that the ApoB/ ApoA1 ratio strongly associated with MS and its components in the Chongqing urban Chinese population, and showed that subjects with MS were 3.5-fold more likely to be in the 75th quartile of the ApoB/ApoA1 ratio (24). However, we found that subjects with MS were 8.13-fold more likely to be in the 75th quartile of the ApoB/ApoA1 ratio, which is more than two times higher than the study mentioned above. These result suggest that the ApoB/ApoA1 ratio appears to be a better predictive marker of MS in PCOS population than that in the other populations.

The association between the ApoB/ApoA1 ratio and each of the MS components was independent of age; however, after adjusting for either BMI or WC together with age, subjects with high FPG and high BP were no longer more likely to be in the high ApoB/ApoA1 group. Our findings indicate that BMI contributed more to the increasing ApoB/ApoA1 ratio than raised FPG and $\mathrm{BP}$ in our subjects. Moreover, WC played a more important role than BMI in increasing the ApoB/ApoA1 ratio.

Hyperandrogenism affects lipid metabolism by the induction of hepatic lipase activity, which is an enzyme that plays a role in the catabolism of HDL particles $(25,26)$. We investigated the relationship between FT, FAI levels and the ApoB/ApoA1 ratio in PCOS patients. Both the high FT group and the high FAI group have higher ApoB/ApoA1 ratio, and there was a statistically significant positive correlation between the ApoB/ApoA1 ratio and FT or FAI; however, the association was no longer significant when we accounted for obesity. Our findings indicate that obesity may contribute more to increasing ApoB/ApoA1 ratio than FT and FAI in this cohort of PCOS patients, which is inconsistent with previous findings (9). This finding suggests that different mechanisms may be involved in assessing CVD risk between PCOS adolescents and adults.

In conclusion, the ApoB/ApoA1 ratio is strongly associated with IR, MS and its components, high FT, and high FAI. A high ApoB/ApoA1 ratio is a valuable predictive marker of MS in the Chinese PCOS adults, and appears to be a better marker in PCOS patients than the other populations. Obesity, especially central obesity, contributes more to increasing ApoB/ApoA1 ratio than raised $\mathrm{BP}, \mathrm{FPG}, \mathrm{FT}$, and FAI in this cohort of PCOS patients.

Despite these relevant findings, it is important to mention the limitations of our study. The present study may be limited by the fact that the cross-sectional study design did not identify the causal relationships between the ApoB/ApoA1 ratio and MS. We look forward to evaluating this issue in future. In addition, all the subjects were from the Gynecological Outpatient Department of Xuzhou Central Hospital, and only included those with complete medical records which may not be representative of the PCOS population in China, and may have selection bias. 


\section{References}

1. Hamilton CA: Low-density lipoprotein and oxidised low-density lipoprotein: their role in the development of atherosclerosis Pharmacol Ther 74: 55-72, 1997.

2. Barter PJ and Rye KA: The rationale for using apoA-I as a clinical marker of cardiovascular risk. J Intern Med 259: 447-454, 2006.

3. Faraj M, Messier L, Bastard JP, Tardif A, Godbout A, Prud'homme D and Rabasa-Lhoret R: Apolipoprotein B: a predictor of inflammatory status in postmenopausal overweight and obese women. Diabetologia 49: 1637-1646, 2006.

4. Franks S: Polycystic ovary syndrome. N Engl J Med 333: 853-861, 1995

5. Maitra A, Pingle RR, Menon PS, Naik V, Gokral JS and Meherji PK: Dyslipidemia with particular regard to apolipoprotein profile in association with polycystic ovary syndrome: a study among Indian women. Int J Fertil Womens Med 46: 271-277, 2001.

6. Sniderman AD, Blank D, Zakarian R, Bergeron J and Frohlich J: Triglycerides and small dense LDL: The twin Achilles heels of the Friedewald formula. Clin Biochem 36: 499-504, 2003.

7. Walldius G, Jungner I, Aastveit AH, Holme I, Furberg CD and Sniderman AD: The apoB/apoA-I ratio is better than the cholesterol ratios to estimate the balance between plasma proatherogenic and antiatherogenic lipoproteins and to predict coronary risk. Clin Chem Lab Med 42: 1355-1363, 2004.

8. McQueen MJ, Hawken S, Wang X, Ounpuu S, Sniderman A, Probstfield J, Steyn K, Sanderson JE, Hasani M, Volkova E, et al; INTERHEART study investigators: Lipids, lipoproteins, and apolipoproteins as risk markers of myocardial infarction in 52 countries (the INTERHEART study): a case-control study. Lancet 372: 224-233, 2008

9. Yin Q, Chen X, Li L, Zhou R, Huang J and Yang D: Apolipoprotein B/apolipoprotein A1 ratio is a good predictive marker of metabolic syndrome and pre-metabolic syndrome in Chinese adolescent women with polycystic ovary syndrome. J Obstet Gynaecol Res 39: 203-209, 2013.

10. Rotterdam ESHRE/ASRM-Sponsored PCOS Consensus Workshop Group: Revised 2003 consensus on diagnostic criteria and long-term health risks related to polycystic ovary syndrome. Fertil Steril 81: 19-25, 2004.

11. Azziz R, Woods KS, Reyna R, Key TJ, Knochenhauer ES and Yildiz BO: The prevalence and features of the polycystic ovary syndrome in an unselected population. J Clin Endocrinol Metab 89: 2745-2749, 2004.

12. Hatch R, Rosenfield RL, Kim MH and Tredway D: Hirsutism: implications, etiology, and management. Am J Obstet Gynecol 140: 815-830, 1981.

13. International Diabetes Federation: The IDF consensus worldwide definition of the metabolic syndrome. http://www.idf. org/webdata/docs/Metabolic_syndrome_definition.pdf. Accessed July 10, 2008.

14. World Health Organization: Measuring obesity: classification and description of anthropometric data: report on a WHO consultation on the epidemiology of obesity, Warsaw, 21-23 October 1987. World Health Organization, Copenhagen, 1989.
15. Chen X, Yang D, Li L, Feng S and Wang L: Abnormal glucose tolerance in Chinese women with polycystic ovary syndrome. Hum Reprod 21: 2027-2032, 2006.

16. Sung KC and Hwang ST: Association between insulin resistance and apolipoprotein B in normoglycemic Koreans. Atherosclerosis 180: 161-169, 2005.

17. Sierra-Johnson J, Romero-Corral A, Somers VK, Lopez-Jimenez F, Walldius G, Hamsten A, Hellénius ML and Fisher RM: ApoB/apoA-I ratio: $\alpha$ independent predictor of insulin resistance in US non-diabetic subjects. Eur Heart J 28: 2637-2643, 2007.

18. Taghibiglou C, Carpentier A, Van Iderstine SC, Chen B, Rudy D, Aiton A, Lewis GF and Adeli K: Mechanisms of hepatic very low density lipoprotein overproduction in insulin resistance. Evidence for enhanced lipoprotein assembly, reduced intracellular ApoB degradation, and increased microsomal triglyceride transfer protein in a fructose-fed hamster model. J Biol Chem 275: 8416-8425, 2000.

19. Taghibiglou C, Rashid-Kolvear F, Van Iderstine SC, Le-Tien H, Fantus IG, Lewis GF and Adeli K: Hepatic very low density lipoprotein-ApoB overproduction is associated with attenuated hepatic insulin signaling and overexpression of protein-tyrosine phosphatase 1B in a fructose-fed hamster model of insulin resistance. J Biol Chem 277: 793-803, 2002.

20. Jing F, Mao Y, Guo J, Zhang Z, Li Y, Ye Z, Ding Y, Wang J, Jin M and Chen K: The value of Apolipoprotein B/Apolipoprotein A1 ratio for metabolic syndrome diagnosis in a Chinese population: a cross-sectional study. Lipids Health Dis 13: 81, 2014.

21. Savas Erdeve S, Simsek E, Dallar Y and Biyikli Z: Utility of ApoB/ApoA1 ratio for the prediction of cardiovascular risk in children with metabolic syndrome. Indian J Pediatr 77: 1261-1265, 2010.

22. Wallenfeldt K, Bokemark L, Wikstrand J, Hulthe J and Fagerberg B: Apolipoprotein B/apolipoprotein A-I in relation to the metabolic syndrome and change in carotid artery intima-media thickness during 3 years in middle-aged men. Stroke 35: 2248-2252, 2004.

23. Sierra-Johnson J, Somers VK, Kuniyoshi FH, Garza CA Isley WL, Gami AS and Lopez-Jimenez F: Comparison of apolipoprotein-B/apolipoprotein-AI in subjects with versus without the metabolic syndrome. Am J Cardiol 98: 1369-1373, 2006.

24. Zhong L, Li Q, Jiang Y, Cheng D, Liu Z, Wang B, Luo R, Cheng $\mathrm{Q}$ and Qing H: The ApoB/ApoA1 ratio is associated with metabolic syndrome and its components in a Chinese population. Inflammation 33: 353-358, 2010.

25. Haffner SM, Kushwaha RS, Foster DM, Applebaum-Bowden D and Hazzard WR: Studies on the metabolic mechanism of reduced high density lipoproteins during anabolic steroid therapy. Metabolism 32: 413-420, 1983.

26. Elbers JM, Giltay EJ, Teerlink T, Scheffer PG, Asscheman H, Seidell JC and Gooren LJ: Effects of sex steroids on components of the insulin resistance syndrome in transsexual subjects. Clin Endocrinol (Oxf) 58: 562-571, 2003. 